\title{
Um Novo Algoritmo IA-RSA Ciente de Imperfeições de Camada Física para Redes Ópticas Elásticas
}

\author{
Alexandre Fontinele, André Soares (Orientador) \\ ${ }^{1}$ Departamento de Computação - Universidade Federal do Piauí (UFPI) \\ Teresina - PI - Brasil \\ alexandrefontineledgmail.com
}

\begin{abstract}
This paper proposes a new Impairment-Aware Routing and Spectrum Assignment (IA-RSA) algorithm for elastic optical networks. The goal of the algorithm is to reduce the blocking probability caused by the degradation of the quality of transmission from new establishments of circuits. Our proposal is compared to two other IA-RSA algorithms: Modified Dijkstra Path Computation $(M D-P C)$ e K-Shortest Path Computation (KS-PC). Simulation results show that the proposed algorithm outperforms the $M D-P C$ and $K S-P C$ algorithms in terms of i) circuit blocking probability, ii) bandwidth blocking probability, iii) fairness of service to different source-destination pairs, and iv) fairness of service to different bandwidths for the EON and NSFNet topologies.
\end{abstract}

Resumo. Este artigo propõe um novo algoritmo de roteamento e alocação de espectro ciente dos efeitos de camada física (IA-RSA - Impairment-Aware Routing and Spectrum Assignment) para redes ópticas elásticas. O objetivo do algoritmo proposto é reduzir a probabilidade de bloqueio causada pela degradação da qualidade de transmissão quando novos circuitos são estabelecidos. $O$ algoritmo proposto é comparado com outros algoritmos IA-RSA: Modified Dijkstra Path Computation (MD-PC) e K-Shortest Path Computation (KS-PC). Resultados de simulação mostram que o algoritmo proposto apresenta um desempenho superior ao dos algoritmos $M D-P C$ e $K S$-PC em termos de i) probabilidade de bloqueio de circuitos, ii) probabilidade de bloqueio de banda, iii) justiça no atendimento de diferentes pares de nós origem e destino e iv) justiça no atendimento de diferentes larguras de banda para as topologias EON e NSFNet.

\section{Introdução}

As soluções de transporte de dados baseadas em rede óptica têm se mostrado promissoras para o futuro das comunicações ópticas de alta velocidade [Chatterjee et al. 2015]. Nas últimas décadas, as soluções de redes ópticas de transporte implantadas e estudadas na literatura eram fortemente baseadas na tecnologia WDM (Wavelength Division Multiplexing) [Nath et al. 2014]. Em uma rede óptica WDM, o espectro é dividido em comprimentos de onda de tamanho fixo [Chatterjee et al. 2015]. A utilização de comprimentos de onda de tamanho fixo provoca a subutilização dos recursos da rede, pois cada requisição de circuito pode requerer diferentes larguras de banda.

Devido à essa deficiência, o uso da tecnologia OFDM (Ortogonal Frequency Division Multiplexing) foi proposto recentemente. Uma rede óptica OFDM tem o potencial de alocar espectro para os circuitos ópticos de acordo com os requisitos de largura de 
banda. Com a tecnologia OFDM o espectro óptico é dividido em slots de frequência $(12,5 \mathrm{GHz})$ e para cada circuito pode ser alocado uma quantidade diferente de slots [Chatterjee et al. 2015]. A utilização do espectro óptico em uma rede óptica OFDM é mais eficiente quando comparada a de uma rede óptica WDM. Essa flexibilidade de alocação de largura de banda das redes ópticas OFDM deu origem a uma nova geração de redes ópticas de transporte, chamada de redes ópticas elásticas.

Para o estabelecimento de um circuito óptico em uma rede óptica elástica é necessário resolver o problema de roteamento e alocação de espectro (RSA - Routing and Spectrum Assignment) [Chatterjee et al. 2015]. O problema RSA consiste em definir uma rota para um par de nós origem e destino. Em seguida, a solução RSA deve escolher uma faixa de espectro livre (conjunto de slots de frequência contíguos) na rota definida para estabelecer o circuito óptico.

O sinal óptico sofre degradação à medida que se propaga da origem para o destino devido às imperfeições da camada física. $\mathrm{O}$ acúmulo das degradações no sinal óptico ao longo de uma rota pode tornar a taxa de erro de bit (BER - Bit Error Rate) intolerável no receptor, inviabilizando a comunicação. Nesse contexto, algoritmos RSA que levam em consideração as imperfeições de camada física (IA-RSA - Impairment-Aware RSA) tornam-se mais apropriados para encontrar uma solução para o problema de roteamento e alocação de espectro.

Este artigo apresenta o trabalho que foi desenvolvido na dissertação [Fontinele 2016]. Em [Fontinele 2016] foi apresentado um novo algoritmo de roteamento e alocação de espectro ciente de imperfeições da camada física em redes ópticas elásticas (IA-RSA). $\mathrm{O}$ algoritmo proposto busca estabelecer novos circuitos reduzindo o impacto na qualidade de transmissão (QoT - Quality of Transmission) dos outros circuitos já ativos na rede. É também apresentado um estudo de avaliação de desempenho comparando o algoritmo proposto com outros dois algoritmos IA-RSA do estado da arte. Os resultados mostram um desempenho superior do algoritmo proposto em relação aos outros algoritmos para diferentes métricas de avaliação.

As demais seções deste artigo estão organizadas da seguinte forma. A Seção 2 discute os trabalhos relacionados e apresenta as contribuições deste artigo. O algoritmo proposto é apresentado na Seção 3. A Seção 4 apresenta um estudo de avaliação de desempenho do algoritmo proposto. Por fim, as conclusões do trabalho são destacadas na Seção 6 .

\section{Trabalhos Relacionados}

A maioria dos trabalhos existentes em redes ópticas elásticas utiliza um limiar de alcance de transmissão [Jinno et al. 2010, Zhou et al. 2012, Gong et al. 2013, Izquierdo-Zaragoza et al. 2014, Chatterjee et al. 2015] relacionado a cada formato de modulação. Também é utilizada uma banda de guarda para evitar a interferência entre circuitos adjacentes. Entretanto, esses métodos podem superestimar ou subestimar os impactos das imperfeições de camada física nas conexões [Zhao et al. 2015].

O conceito de algoritmos RSA cientes de imperfeições de camada física (IA-RSA) é importante devido aos efeitos prejudiciais da coexistência de vários circuitos ópticos com diferentes formatos de modulações e taxas de bits [Beyranvand and Salehi 2013]. 
Também é importante escolher o formato de modulação adequado para um circuito através de algoritmos RSA cientes de qualidade transmissão [Beyranvand and Salehi 2013, Chatterjee et al. 2015].

Os autores em [Beyranvand and Salehi 2013] propõem um esquema de RSA ciente da qualidade de transmissão (QoT) para redes ópticas elásticas. O esquema emprega três etapas: i) cálculo das rotas viáveis, ii) seleção de uma rota e iii) alocação de espectro. O artigo apresenta um modelo analítico para calcular o SNR (Signal to Noise Ratio) de uma dada rota. Baseado no esquema proposto são apresentados dois algortimos IA-RSA: Modified Dijkstra Path Computation (MD-PC) e K-Shortest Path Computation (KS-PC).

Algoritmos RSA cientes de QoT devem garantir a qualidade do sinal de um circuito em estabelecimento sem interromper os circuitos já presentes na rede. Essa preocupação com a QoT dos circuitos já ativos na rede ainda é pouco estudada em redes ópticas elásticas [Chatterjee et al. 2015, Zhao et al. 2015]. A principal contribuição deste artigo é a proposta de um novo algoritmo IA-RSA para redes ópticas elásticas sob tráfego dinâmico. O principal diferencial do algoritmo proposto em relação às outras propostas presentes na literatura são as estratégias utilizadas para as escolhas do formato de modulação e da rota para cada circuito óptico em estabelecimento. Essas estratégias buscam estabelecer circuitos mais resistentes às degradações dos efeitos de camada física e que reduzam o impacto na QoT dos outros circuitos já ativos na rede.

\section{Algoritmo Proposto}

O algoritmo IA-RSA proposto neste trabalho é chamado de $k$ menores caminhos com redução de QoTO (KSP-RQoTO - K-Shortest Path with Reduction of QoTO). O algoritmo computa os $k$ menores caminhos para cada par origem e destino da rede em uma fase offline. No momento que a rede está em operação, o algoritmo proposto busca alocar uma das $k$ rotas alternativas de forma a minimizar o bloqueio do tipo QoTO (QoT inadequada para os outros circuitos ópticas já ativos na rede). O fluxograma ilustrado na Figura 1 apresenta os passos seguidos pelo algoritmo proposto na fase de operação da rede.

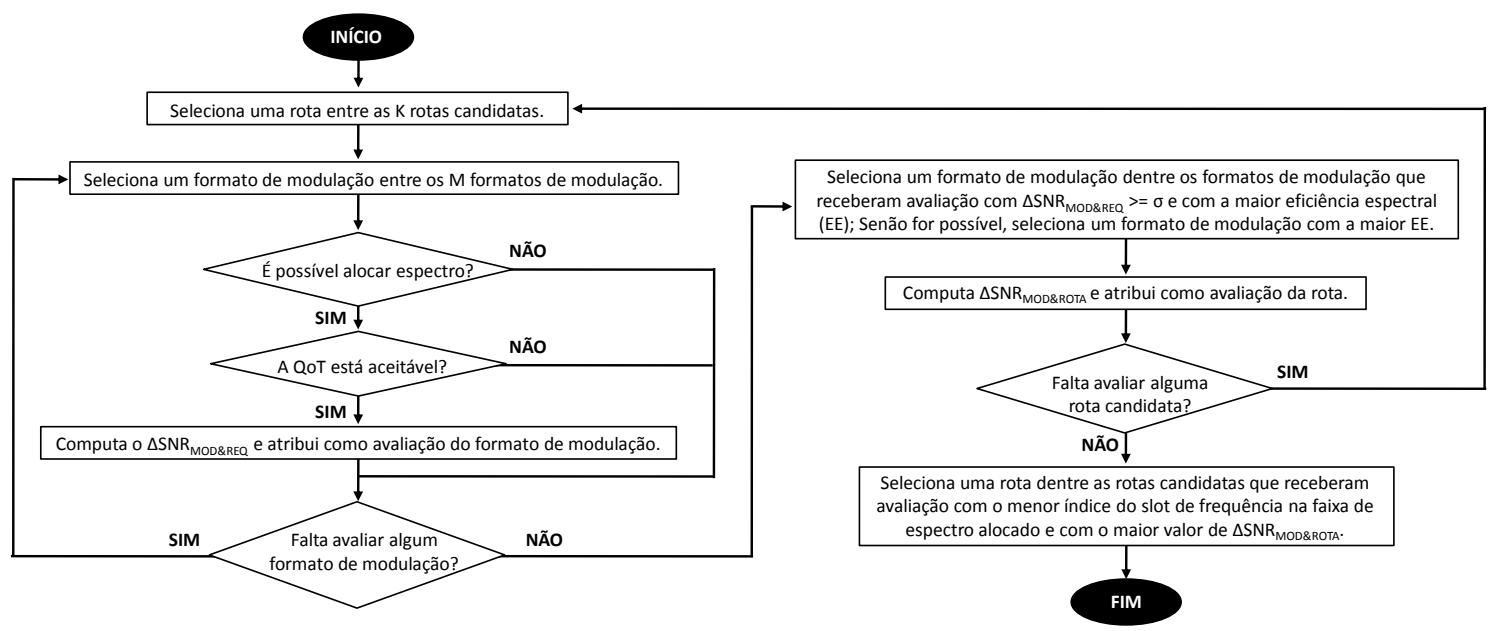

Figura 1. Fluxograma do algoritmo KSP-RQoTO.

O primeiro passo do algoritmo KSP-RQoTO é selecionar um formato de modulação para cada rota candidata. O parâmetro $\sigma$ é utilizado para ajudar a buscar 
por formatos de modulação que deixem os circuitos mais resistentes a interferências de outros circuitos. A escolha do valor ideal de $\sigma$ deve ser feita durante a fase de planejamento da rede ou durante uma alteração da configuração da rede. O segundo passo do algoritmo KSP-RQoTO é selecionar uma rota entre as rotas candidatas. O algoritmo tenta escolher rotas que reduzam a fragmentação do espectro e que causem menos degradações nos circuitos já ativos. Mais detalhes sobre o funcionamento do algoritmo KSP-RQoTO podem ser encontrados na Seção 4.1 em [Fontinele 2016].

\section{Avaliação de Desempenho}

O algoritmo proposto, KSP-RQoTO, foi comparado com os algoritmos KS-PC e MD-PC sob os aspectos de i) probabilidade de bloqueio de circuito, ii) probabilidade de bloqueio de banda, iii) justiça no atendimento de diferentes pares de nós origem e destino e iv) justiça no atendimento de diferentes larguras de banda. Foram utilizadas quatro rotas candidatas para os algoritmos de k menores caminhos. O algoritmo First-Fit foi utilizado para a alocação de espectro. Devido ao limite de páginas serão apresentados apenas os resultados de probabilidade de bloqueio de circuito e justiça no atendimento de diferentes pares de nós origem e destino. Os demais resultados e mais detalhes sobre os cenários das simulações podem ser encontrados na Seção 4.2 em [Fontinele 2016].

A Figura 2 apresenta as probabilidades de bloqueio de circuitos obtidas com os algoritmos KSP-RQoTO, KS-PC e MD-PC em função da carga na rede para as topologias EON e NSFNet. O valor entre parênteses na legenda do algoritmo KSP-RQoTO, ilustrada na Figura 2, corresponde ao valor ideal para o $\sigma$. Observando a Figura 2, nota-se que, sob os valores de carga analisados, o algoritmo KSP-RQoTO apresentou melhor desempenho em termos de probabilidade de bloqueio de circuitos do que os algoritmos KS-PC e MD$\mathrm{PC}$ para as duas topologias consideradas.

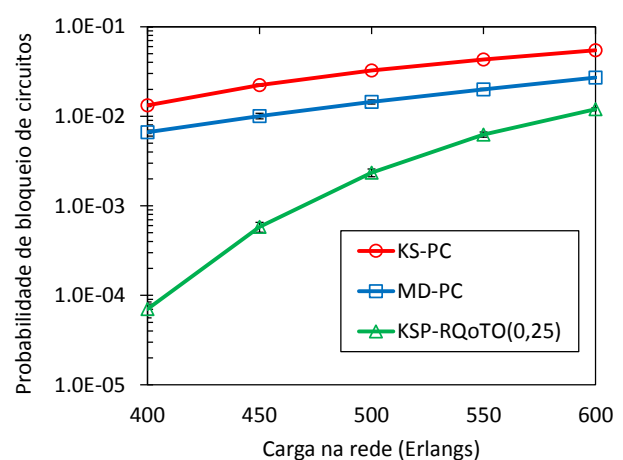

(a) EON.

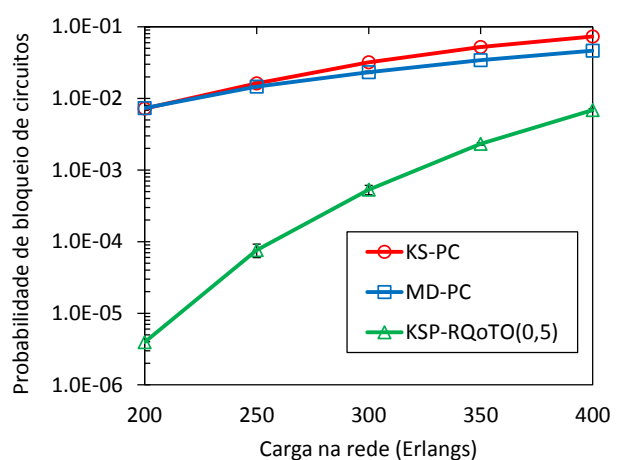

(b) NSFNet.

Figura 2. Probabilidades de bloqueio de circuito obtidas pelos algoritmos KS-PC, MD-PC e KSP-RQoTO paras as topologias (a) EON e (b) NSFNet.

Sob uma carga de 600 Erlangs na topologia EON, Figura 2(a), os algoritmos KSPC, MD-PC e KSP-RQoTO obtiveram 5,48\%, 2,71\% e 1,20\% de probabilidade de bloqueio de circuito, respectivamente. Para esses valores de probabilidades de bloqueio de circuito, o ganho do KSP-RQoTO em relação aos algoritmos KS-PC e MD-PC foi de aproximadamente 78,10\% e 55,75\%, respectivamente. Sob uma carga de 400 Erlangs na topologia NSFNet, Figura 2(b), os algoritmos KS-PC, MD-PC e KSP-RQoTO alcançaram 
$7,30 \%, 4,63 \%$ e $0,69 \%$ de probabilidade de bloqueio de circuito, respectivamente. O ganho do algoritmo KSP-RQoTO em relação aos algoritmos KS-PC e MD-PC foi de aproximadamente $90,59 \%$ e $85,18 \%$, respectivamente.

A Figura 3 mostra o desempenho dos algoritmos KS-PC, MD-PC e KSP-RQoTO em termos do desvio padrão entre as probabilidades de bloqueio de circuito para cada par de nós origem e destino nas topologias EON e NSFNet. Como pode ser observado na Figura 3, o algoritmo KSP-RQoTO mostra um menor desvio padrão quando comparado aos outros algoritmos analisados para ambas as topologias consideradas. Isto significa que o algoritmo KSP-RQoTO apresenta um maior nível de justiça no atendimento de diferentes pares de nós origem e destino na rede quando aplicado nas topologias EON e NSFNet em relação aos algoritmo KS-PC e MD-PC.

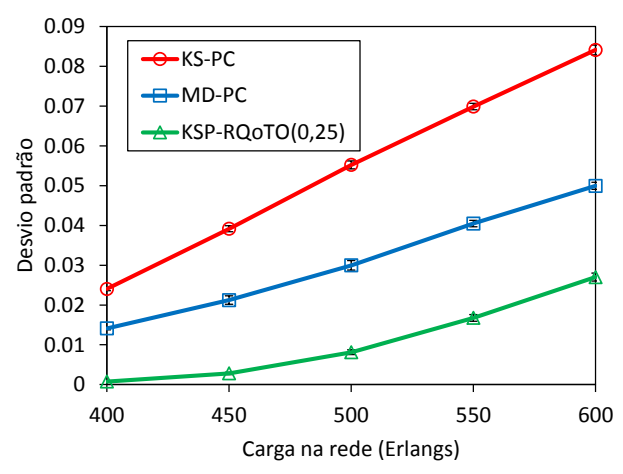

(a) EON.

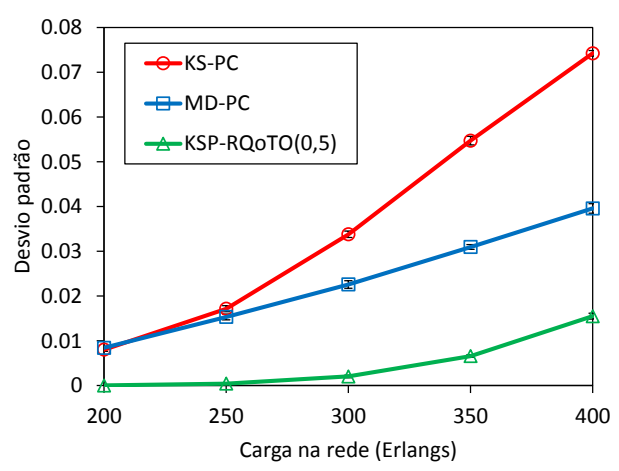

(b) NSFNet.

Figura 3. Desvio padrão entre as probabilidades de bloqueio de circuitos obtidas para cada par de nós origem e destino para os algoritmo KS-PC, MD-PC e KSPRQoTO nas topologias (a) EON e (b) NSFNet.

\section{Publicações}

As soluções propostas ao longo do mestrado, que geraram a dissertação, e que foram publicadas podem ser encontradas na Seção 1.5 da dissertação [Fontinele 2016]. As publicações mais recentes que contém os resultados apresentados na dissertação foram no SBRC 2016 (Qualis B2) [Fontinele et al. 2016] e na revista Computer Networks (Qualis A1) [Fontinele et al. 2017].

\section{Conclusões}

Este artigo resumiu a contribuição da dissertação de mestrado [Fontinele 2016], que estudou roteamento e alocação de espectro ciente dos efeitos de camada física em redes ópticas elásticas. Foi apresentado o algoritmo KSP-RQoTO, que tem como objetivo estabelecer circuitos que causem o menor impacto na QoT dos outros circuitos já ativos na rede.

O algoritmo KSP-RQoTO apresentou um desempenho superior ao dos algoritmos KS-PC e MD-PC em termos de i) probabilidade de bloqueio de circuito, ii) probabilidade de bloqueio de banda, iii) justiça no atendimento de diferentes pares de nós origem e destino e iv) justiça no atendimento de diferentes larguras de banda para as topologias EON e NSFNet. 
Em geral, em termos de probabilidade de bloqueio de circuitos, o algoritmo proposto apresenta um ganho mínimo de 78,10\% e 55,75\% quando comparado aos algoritmos KS-PC e MD-PC, respectivamente. Além disso, o algoritmo proposto apresenta um desempenho superior quando comparado aos algoritmos KS-PC e MD-PC em termos de justiça.

\section{Referências}

Beyranvand, H. and Salehi, J. (2013). A quality-of-transmission aware dynamic routing and spectrum assignment scheme for future elastic optical networks. Journal of Lightwave Technology, 31(18):3043-3054.

Chatterjee, B. C., Sarma, N., and Oki, E. (2015). Routing and spectrum allocation in elastic optical networks: A tutorial. IEEE Communications Surveys Tutorials, 17(3):17761800 .

Fontinele, A. (2016). Um Novo Algoritmo RSA Ciente de Imperfeições de Camada Física para Redes Ópticas Elásticas. Universidade Federal do Piauí, Teresina.

Fontinele, A., Santos, I., Neto, J. N., Campelo, D. R., and Soares, A. (2017). An efficient ia-rmlsa algorithm for transparent elastic optical networks. Computer Networks, 118:1 -14 .

Fontinele, A., Santos, I., Nolêto-Neto, J., Campelo, D., and Soares, A. (2016). Um novo algoritmo rsa ciente de imperfeições de camada física para redes ópticas elásticas. In XXXIV Simpósio Brasileiro de Redes de Computadores e Sistemas Distribuídos (SBRC), pages 322-335.

Gong, L., Zhou, X., Liu, X., Zhao, W., Lu, W., and Zhu, Z. (2013). Efficient resource allocation for all-optical multicasting over spectrum-sliced elastic optical networks. IEEE/OSA Journal of Optical Communications and Networking, 5(8):836-847.

Izquierdo-Zaragoza, J.-L., Pavon-Marino, P., and Bueno-Delgado, M.-V. (2014). Distance-adaptive online rsa algorithms for heterogeneous flex-grid networks. In 2014 International Conference on Optical Network Design and Modeling, pages 204-209.

Jinno, M., Kozicki, B., Takara, H., Watanabe, A., Sone, Y., Tanaka, T., and Hirano, A. (2010). Distance-adaptive spectrum resource allocation in spectrum-sliced elastic optical path network [topics in optical communications]. IEEE Communications Magazine, 48(8):138-145.

Nath, I., Chatterjee, M., and Bhattacharya, U. (2014). A survey on regenerator placement problem in translucent optical network. In 2014 International Conference on Circuits, Systems, Communication and Information Technology Applications (CSCITA), pages 408-413.

Zhao, J., Wymeersch, H., and Agrell, E. (2015). Nonlinear impairment aware resource allocation in elastic optical networks. In 2015 Optical Fiber Communications Conference and Exhibition (OFC), pages 1-3.

Zhou, X., Lu, W., Gong, L., and Zhu, Z. (2012). Dynamic rmsa in elastic optical networks with an adaptive genetic algorithm. In 2012 IEEE Global Communications Conference (GLOBECOM), pages 2912-2917. 